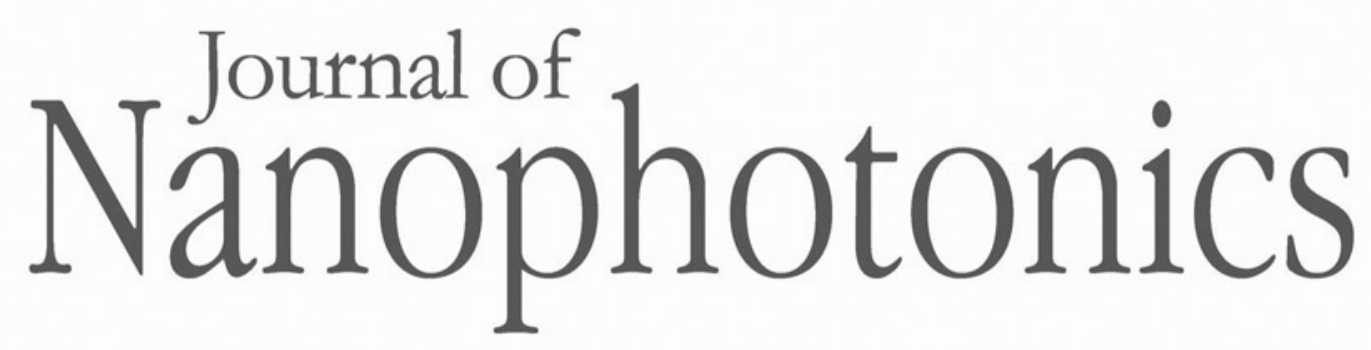

Nanophotonics.SPIEDigitalLibrary.org

\title{
Investigation of indium gallium nitride facet-dependent nonpolar growth rates and composition for core-shell light-emitting diodes
}

Ionut Gîrgel

Paul R. Edwards

Emmanuel Le Boulbar

Pierre-Marie Coulon

Suman-Lata Sahonta

Duncan W. E. Allsopp

Robert W. Martin

Colin J. Humphreys

Philip A. Shields 


\title{
Investigation of indium gallium nitride facet-dependent nonpolar growth rates and composition for core-shell light-emitting diodes
}

\author{
Ionut Gîrgel, ${ }^{\text {a }}$ Paul R. Edwards, ${ }^{\mathrm{b}}$ Emmanuel Le Boulbar, ${ }^{\mathrm{a}}$ \\ Pierre-Marie Coulon, ${ }^{a}$ Suman-Lata Sahonta, ${ }^{c}$ Duncan W. E. Allsopp, \\ Robert W. Martin, ${ }^{b}$ Colin J. Humphreys, ${ }^{c}$ and Philip A. Shields ${ }^{\mathrm{a}} *$ \\ ${ }^{a}$ University of Bath, Department of Electronic and Electrical Engineering, Claverton Down, \\ Bath BA2 7AY, United Kingdom \\ ${ }^{b}$ University of Strathclyde, Department of Physics, SUPA, 107 Rottenrow, \\ Glasgow G4 0NG, United Kingdom \\ ${ }^{c}$ University of Cambridge, Department of Materials Science and Metallurgy, \\ 27 Charles Babbage Road, Cambridge CB3 OFS, United Kingdom
}

\begin{abstract}
Core-shell indium gallium nitride $(\mathrm{InGaN}) / \mathrm{gallium}$ nitride $(\mathrm{GaN})$ structures are attractive as light emitters due to the large nonpolar surface of rod-like cores with their longitudinal axis aligned along the c-direction. These facets do not suffer from the quantum-confined Stark effect that limits the thickness of quantum wells and efficiency in conventional light-emitting devices. Understanding InGaN growth on these submicron three-dimensional structures is important to optimize optoelectronic device performance. In this work, the influence of reactor parameters was determined and compared. GaN nanorods (NRs) with both $\{11-20\} a$-plane and \{10-10\} $\mathrm{m}$-plane nonpolar facets were prepared to investigate the impact of metalorganic vapor phase epitaxy reactor parameters on the characteristics of a thick (38 to $85 \mathrm{~nm}$ ) overgrown InGaN shell. The morphology and optical emission properties of the InGaN layers were investigated by scanning electron microscopy, transmission electron microscopy, and cathodoluminescence hyperspectral imaging. The study reveals that reactor pressure has an important impact on the InN mole fraction on the $\{10-10\} m$-plane facets, even at a reduced growth rate. The sample grown at $750^{\circ} \mathrm{C}$ and 100 mbar had an InN mole fraction of $25 \%$ on the $\{10-10\}$ facets of the NRs. (c) The Authors. Published by SPIE under a Creative Commons Attribution 3.0 Unported License. Distribution or reproduction of this work in whole or in part requires full attribution of the original publication, including its DOI. [DOI: 10.1117/1.JNP.10.016010]
\end{abstract}

Keywords: core-shell; indium gallium nitride; $m$-plane; $a$-plane; nonpolar; cathodoluminescence.

Paper 15161P received Nov. 6, 2015; accepted for publication Feb. 11, 2016; published online Mar. 7, 2016.

\section{Introduction}

Most commercial blue light-emitting diodes (LEDs) use indium gallium nitride (InGaN)/gallium nitride $(\mathrm{GaN})$ quantum wells $(\mathrm{QWs})$ in the active region, grown in the [0001] direction, i.e., parallel to the $c$-plane. These have inherent limitations due to built-in electric fields along the [0001] direction, which lead to reduced electron-hole overlap in the active QWs as a result of the quantum-confined Stark effect ${ }^{1}$ (QCSE).

Avoiding the QCSE in polar materials normally requires the QWs to be grown perpendicular to the polar axis on either the nonpolar $\{11-20\}$ and $\{10-10\}$ planes in wurtzite materials. Another option in the III-nitrides is to grow InGaN QWs on semipolar crystallographic planes, in particular, planes oriented at or close to an angle of $\sim 45$ deg to the polar axis. ${ }^{1}$ However, growth of III-nitrides on such nonpolar and semipolar planes is less mature than for the (0001) plane, with $\{10-10\}$ growth typically requiring bulk substrates to avoid high levels of stacking fault defects. ${ }^{2}$

*Address all correspondence to: Philip A. Shields, E-mail: p.shields@bath.ac.uk 
In this respect, core-shell ${ }^{3-5} \mathrm{InGaN} / \mathrm{GaN}$ nanorods (NRs) are of considerable interest due to their large surface area of nonpolar planes or facets with lower number of defects, ${ }^{6}$ the potential for high surface to volume ratio, ${ }^{7}$ and QWs grown on their nonpolar sidewalls not being subject to the detrimental QCSE. These reasons provide a strong motivation for investigating their growth by the commercially preferred metalorganic vapor phase epitaxy (MOVPE) method. Such NRs can be grown using either a bottom-up approach using selective area epitaxy ${ }^{8-14}$ or a top-down approach ${ }^{15,16}$ in which NRs with controlled aspect ratio are etched from a planar film before the regrowth of GaN/InGaN shell layers over the NRs. ${ }^{4,16,17}$ Irrespective of their method of formation, there is substantial evidence that GaN NRs are strain-free once their height exceeds their diameter. ${ }^{17,18}$ Therefore, the only strain in the core-shell structures described here will be due to lattice mismatch between InGaN and relaxed GaN. A particular advantage of the top-down approach lies in its potential to be highly uniform on a wafer scale. ${ }^{15,18,19}$

In contrast to planar layer growth, uniform InGaN growth on NRs is difficult because the three-dimensional (3-D) growth mode leads to facet-dependent growth rates and indium nitride (InN) incorporation, leading to emission at multiple peak wavelengths. ${ }^{4,5,17,20}$ Further, InGaN growth on pre-etched GaN NRs can be nonuniform for closely packed arrays, ${ }^{12,21}$ indicating a likely dependence of indium mole fraction incorporation on NR height and spacing.

In this paper, we report the growth of InGaN layers on etched NR arrays after the crystallographic facets has been recovered by an initial $\mathrm{GaN}$ growth step. The emission properties of ensuing InGaN layers grown under different conditions are compared, in order to understand which parameters significantly influence the $\mathrm{InGaN}$ growth on the different facets available on the GaN NRs. This information is critical for optimizing the manufacture of core-shell QW structures for solid state lighting. ${ }^{22}$

\section{Experiments}

\subsection{Fabrication of Template for Metalorganic Vapor Phase Epitaxy Regrowth}

NR arrays were created with a top-down approach ${ }^{15}$ from a planar GaN/AlN/Si(111) wafer. A pattern of nickel dots, obtained by nanoimprint lithography and a liftoff process,$^{23}$ was used to make a hard mask for a highly anisotropic $\mathrm{Cl}_{2} / \mathrm{Ar}$ plasma etch to form the array of GaN NR cores. The pattern consisted of a hexagonal array of $\mathrm{Ni}$ dots and, after the top-down plasma etch process, NRs of $\sim 700 \mathrm{~nm}$ diameter and a pitch of $2 \mu \mathrm{m}$ resulted, etched completely through the $\mathrm{GaN}$, the AlN buffer layer, and $200 \mathrm{~nm}$ into the $\mathrm{Si}(111)$ substrate.

Figure 1(a) shows a schematic of the GaN NRs fabricated by this process. The starting undoped $\mathrm{GaN}$ film was $1.5-\mu \mathrm{m}$ thick with an additional $0.5-\mu \mathrm{m}$ buffer layer of AlN used to manage the lattice strain to the GaN. Etching the NRs down into the Si substrate has the advantage that the etched $\mathrm{Si}$ surface can be converted to form a $\mathrm{SiN}_{x}$ selective growth mask, as seen in Fig. 1(a), by an in-situ nitridation step prior to the $\mathrm{GaN}$ facet recovery step. This leads to GaN regrowth only on the etched GaN cores with negligible if any growth on the exposed AlN and $\mathrm{Si}$ surfaces, as shown in scanning electron microscope (SEM) images in Figs. 1(b) and 1(c). ${ }^{24}$

\subsection{Metalorganic Vapor Phase Epitaxy Regrowth}

After the nitridation step, performed at $1050^{\circ} \mathrm{C}$ for $10 \mathrm{~min}$ and a short $\mathrm{GaN}$ nucleation step, a $\mathrm{GaN}$ facet recovery step was performed in the MOVPE reactor at $1060^{\circ} \mathrm{C}$ and $50 \mathrm{mbar}$ for $5 \mathrm{~min}$ in order to obtain NRs with a regular, faceted shape, notably flat nonpolar facets. These growth conditions were chosen to retain as many crystal planes as possible, in particular, the $\{11-20\}$ plane, as shown in Fig. 1(b). In this work, for the subsequent InGaN layer growth, the reactor parameters rather than the NR spacing ${ }^{17}$ determine the relative facet growth rates and the InN fraction incorporation on the respective facets. The TMIn and TMGa flows were kept constant across all the InGaN growths at 200 and $9 \mathrm{sccm}$, respectively. Three different InGaN growth conditions were considered with the following parameters: $750^{\circ} \mathrm{C}$ at $300 \mathrm{mbar}, 700^{\circ} \mathrm{C}$ at $300 \mathrm{mbar}$, and $750^{\circ} \mathrm{C}$ at $100 \mathrm{mbar}$, on the same faceted GaN scaffold [Fig. 1(b)], while all other parameters were unchanged. A growth time of $30 \mathrm{~min}$ was used in order to determine 

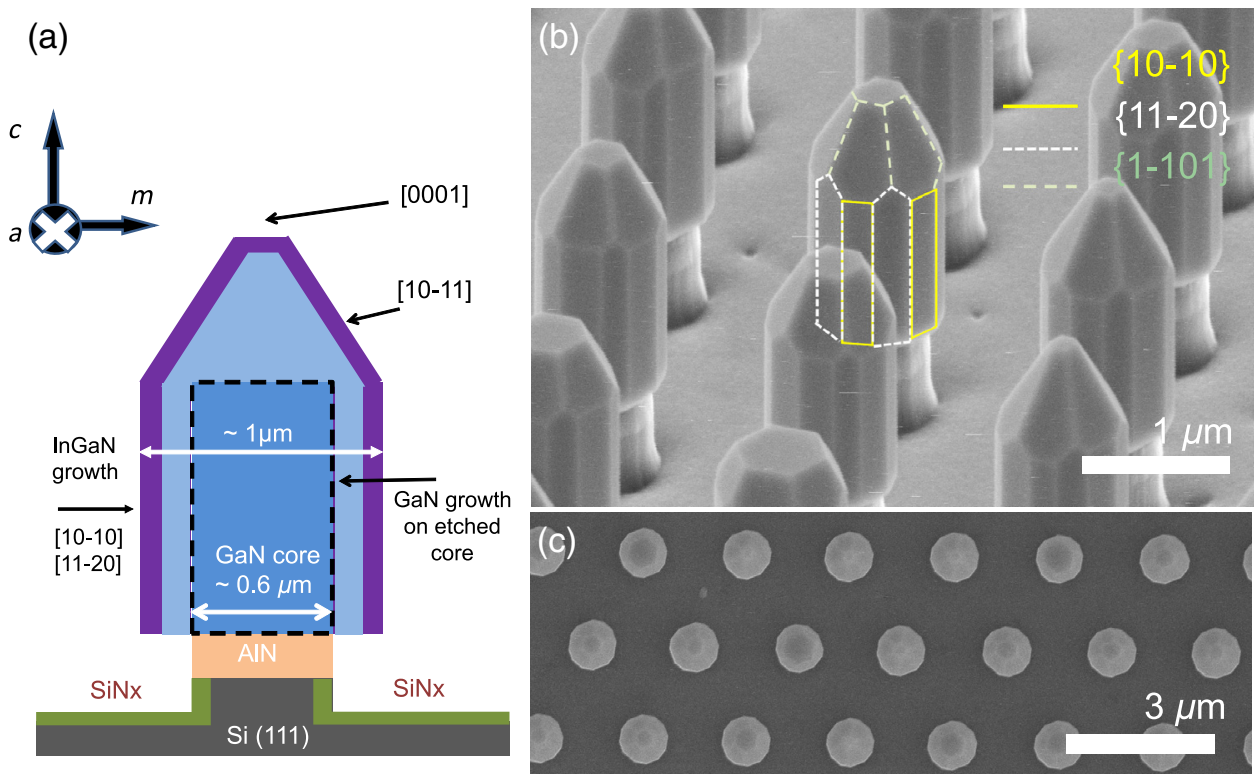

Fig. 1 (a) Schematic of the cross-section of a NR after GaN regrowth on the initial etched GaN core (not drawn to scale), (b) tilted secondary-electron microscope (SEM) image of NRs with smooth nonpolar facets and a small remaining flat $c$-plane, and (c) plan view NR array with 2- $\mu \mathrm{m}$ pitch.

reliable growth rates from measurements of the thickness of the nonpolar InGaN via SEM images, especially for structures grown at temperatures $\left(700\right.$ to $\left.800^{\circ} \mathrm{C}\right)$ that encourage InGaN desorption ${ }^{25}$ i.e., when the $\mathrm{InN}$ incorporation rate is low.

\section{Characterization of Indium Gallium Nitride Layers}

The morphology of the InGaN layers was examined by taking planar secondary-electron microscope images of arrays of over 80 NRs for each image, and an example can be seen in Fig. 2(a). ${ }^{24}$ Image processing software was used to attain statistically meaningful conclusions about the amount of growth after each of the growth steps. The SEM magnification and the image processing algorithm were kept unchanged, and only complete NRs were considered. The standard deviation of the NR equivalent diameters was $6 \mathrm{~nm}$ on the etched GaN, $12 \mathrm{~nm}$ on regrown $\mathrm{GaN}$, and between 13 and $17 \mathrm{~nm}$ for the different InGaN growth conditions. The effect of the different growth conditions on diameter distributions is shown in Fig. 2(b).

(a)

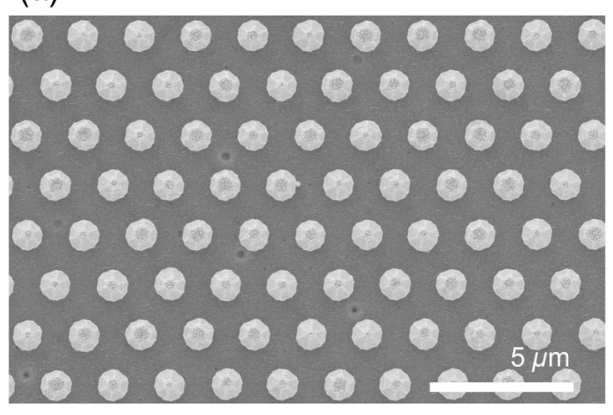

(b)

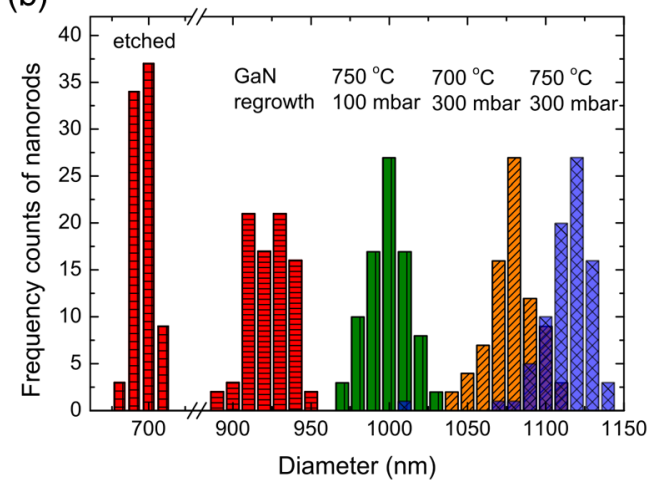

Fig. 2 (a) SE image showing a large array of NRs used to determine equivalent diameters from the InGaN growth at $700^{\circ} \mathrm{C}$ and 300 mbar sample and (b) evolution of NR diameters from GaN etching and regrowth for the three growth conditions used for InGaN. 
One sample $\left(700^{\circ} \mathrm{C}, 300 \mathrm{mbar}\right)$ was analyzed by transmission electron microscopy (TEM) to examine the InGaN growth in more detail. ${ }^{24}$ The optical properties were assessed by cathodoluminescence (CL) hyperspectral imaging to determine emission characteristics, notably uniformity on the nonpolar facets of interest and to estimate $\mathrm{InN}$ mole fraction. ${ }^{26}$

The GaN NRs retained a small $c$-plane [Fig. 1(b)] after the short GaN facet recovery growth. With sufficient growth time, the NR tips would form into a self-limiting pyramid shape ${ }^{27-29}$ with the slower growing and more stable semipolar $\{1-101\}$ facets causing extinction of the faster growing planes ${ }^{30}$ at convex surfaces. Similarly, extinction of the faster growing $a$-planes will occur on the vertical sidewalls of the NRs, leaving structures of hexagonal cross-section, terminated by $m$-planes. Since InGaN growth on the $a$-planes formed part of this study, the duration of the GaN facet recovery growth was kept short, with the consequence the NRs had a residual [0001] plane. Figures 3(a)-3(c) show secondary electron SEM images of the results of the three InGaN growth runs in which various crystal orientations are retained and can be identified: [0001], [1-101], [11-20], and [10-10]. In contrast to GaN regrowth, poor selectivity was observed during InGaN deposition, as $\mathrm{InGaN}_{\text {growth }}$ can be seen on the $\mathrm{SiN}_{x}$ surface in Figs. 3(a)-3(c). On the NRs, increased surface roughness of the $c$-plane is observed, indicating a layer of unstrained indium rich growth. ${ }^{31,32}$

On the macroscale, the different crystal planes are known to have different relative growth rate $^{30}$ determined by growth parameters ${ }^{33,34}$ and potentially result in different $\mathrm{InN}$ mole fractions on the crystal planes, as shown by previous work. ${ }^{4,17,29}$ However, it is unclear if the growth dynamics on rod-shaped nanostructures will be the same. For example, the dimensions of the facets could be less than the surface diffusion length of Ga or In, and pseudomorphic strain at the interfaces between planes may be lower. Both factors could influence the InN fraction. On the NRs used in this work, the $\{11-20\}$ and $\{10-10\}$ facets originating from the template are retained after the InGaN growth.

The SEM images in Fig. 3 show that changes in growth parameters and the long growth time had a clear impact on the morphology of the crystal facets, in particular, the relative growth rates
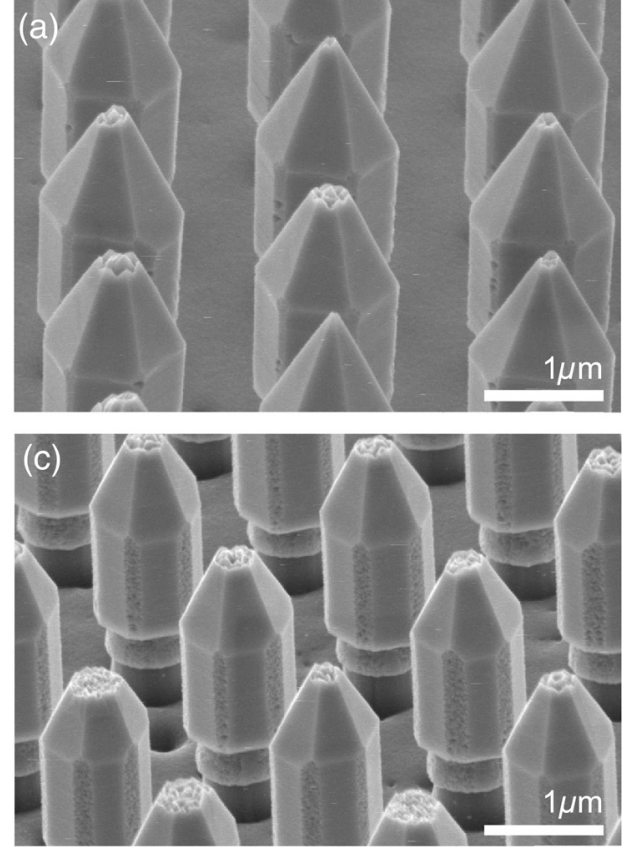
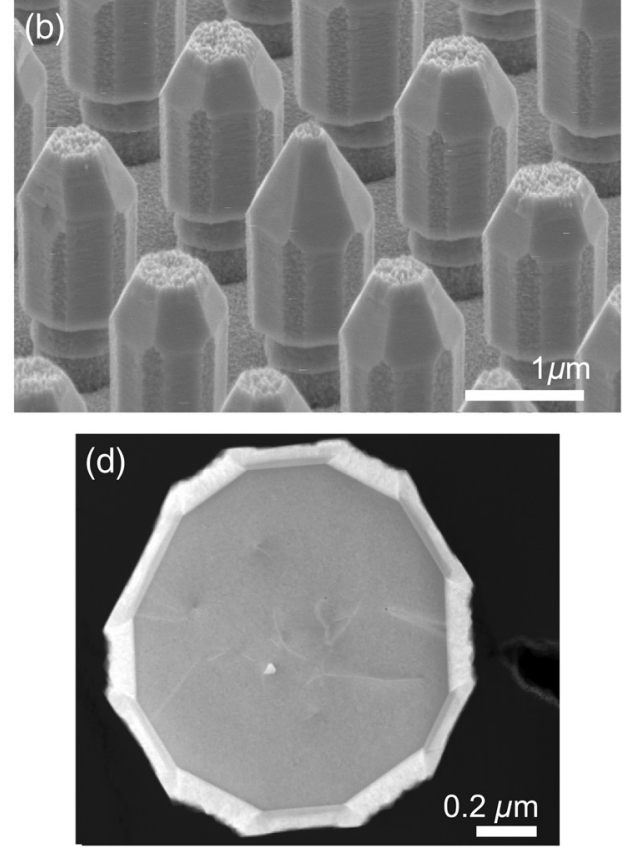

Fig. 3 NRs after InGaN layer growth: (a) $750^{\circ} \mathrm{C} 300$ mbar shows a small remaining (0001) plane with nanopyramids, $\{10-10\} \mathrm{m}$-planes, and minimized $\{11-20\}$ facets; (b) $700^{\circ} \mathrm{C}$ and $300 \mathrm{mbar}$ results in an increase in roughness on all planes; (c) $750^{\circ} \mathrm{C}$ and $100 \mathrm{mbar}$ shows nanopyramids on the (0001) $c$-plane, $m$-planes more similar to the high temperature growth, and high roughness on the $\{11-20\}$ a-plane, more similar to the low temperature sample; (d) TEM lamella of sample obtained at $700^{\circ} \mathrm{C}$ and 300 mbar shows different lateral growth thickness and length between the $a$ - and $m$-planes. 
Gîrgel et al.: Investigation of indium gallium nitride facet-dependent nonpolar growth rates...

Table 1 Comparison of growth rates corresponding to the three InGaN growths.

\begin{tabular}{lcccc}
\hline \hline Sample & Technique & $\begin{array}{c}\text { Diameter } \\
(\text { Std dev }) \\
(\mathrm{nm})\end{array}$ & $\begin{array}{c}\text { Radius increase- } \\
\text { layer thickness } \\
(\mathrm{nm})\end{array}$ & $\begin{array}{c}\text { Average growth rate } \\
\text { of nonpolar facets } \\
(\mathrm{nm} / \mathrm{min})\end{array}$ \\
\hline GaN regrowth & SEM & $923(13)$ & & \\
$750^{\circ} \mathrm{C} 300 \mathrm{mbar}$ & SEM & $1113(17)$ & 95.2 & 3.2 \\
$750^{\circ} \mathrm{C} 100 \mathrm{mbar}$ & SEM & $999(13)$ & 37.9 & 1.3 \\
$700^{\circ} \mathrm{C} 300 \mathrm{mbar}$ & SEM & $1079(15)$ & 78.0 & 2.6 \\
& TEM & 1265 & 84.6 & 2.8 \\
\hline \hline
\end{tabular}

of the facets, ${ }^{34}$ which in turn are related to the surface energies of the various planes. ${ }^{35,36}$ The growth rates shown in Table 1 were obtained from the increase in equivalent diameter, as determined from the image analysis software.

Figures 3(a)-3(c) show that temperature and pressure have the dominant impact on the $a$-and the $m$-planes, as far as determining the relative size and their surface roughness. Use of a high temperature has reduced the proportion of $a$ - to $m$-plane observable compared with the facetted $\mathrm{GaN}$ core because the temperature increases the relative growth rate of the $\{11-20\}$ facet, and thus, this sample has only a small residual $\{11-20\}$ surface. Furthermore, there is a tendency for indium atoms to stick at the apices between planes, which create more irregular surfaces where the adatoms can be captured, leading to nonuniform-wavelength light emission. ${ }^{17}$ At $700{ }^{\circ} \mathrm{C}$ and $300 \mathrm{mbar}$, the relative growth rates of the nonpolar planes are closer in value, causing a different $m$ - to $a$-plane growth rate ratio. Also the indium desorption and diffusion length are both lower than at $750^{\circ} \mathrm{C}$, making the incorporation of more indium atoms possible. As the indium atom diffusion length becomes comparable to the distance between binding sites on the sidewalls, there is a visible roughening of the sidewalls. In Ref. 37, it is indicated that a statistical roughening of the surfaces can be expected on the (0001) and (000-1) surfaces if the diffusion length becomes shorter than the mean distance between binding sites. Surface roughening encourages local fluctuations in the InN mole fraction on the nonpolar planes. Reduction of the growth pressure to 100 mbar, while keeping the temperature at $750^{\circ} \mathrm{C}$, had the effect of reducing the InGaN growth rates of both the $m$ - and $a$-planes, to an average rate of $\sim 1.3 \mathrm{~nm} / \mathrm{min}$, as the increase in the size of the overall NR is smaller. The presence of residual $\{11-20\} a$-planes on this sample indicates that the relative growth rates of the $m$ - to $a$-plane differ from the other sample grown at $750^{\circ} \mathrm{C}$. Note the reduction in growth pressure tends to increase indium surface diffusion, ${ }^{38}$ because low pressure reduces the indium atom collision probability. In support of this, the roughness has diminished on the $\{10-10\} m$-plane in Fig. 3(c) compared to Fig. 3(b), while the $\{11-20\} a$-plane is still very rough even at low growth rate. The size of the $a$-plane is determined by the initial GaN template, where high temperature and low pressure reduce the relative growth rate difference between the $a$ - and $m$-planes. With the range of growth parameters available, the $a$-plane grows as fast as or faster than the $m$-plane, suggesting that finding a growth recipe to create a substantial $a$-plane surface area would be difficult to achieve for a core-shell device.

Table 1 lists the combined average growth rates of the nonpolar planes obtained from the image analysis method for the three samples used in this work. The growth rates were determined from SEM images and from a TEM image of the radial cross-section (i.e., plan view) of a single NR. The estimates of growth rates from SEM measurements were determined by statistical analysis of a set of NRs, on equipment calibrated differently than the TEM, which accounts for the discrepancy in diameter of the same sample. Image processing for the TEM data was done in a similar manner to the SEM data. The growth rate was determined to be $2.3 \mathrm{~nm} / \mathrm{min}$ on the $m$-plane, and $3.1 \mathrm{~nm} / \mathrm{min}$ on the $a$-plane, making the ratio of $m$-plane to $a$-plane growth rates 0.74 , for $700^{\circ} \mathrm{C}$ and 300 mbar.

In examining the TEM lamella in Fig. 3(d), it is worth mentioning that the GaN facets grow seamlessly at the etched interfaces, because there is no difference in contrast between the core 
and regrown facets. However, the InGaN shell is immediately distinguishable. Furthermore, the different contrast at the $m$-plane interfaces indicates a possible change in InN mole fraction after an initial layer thickness $(\sim 20 \mathrm{~nm})$ is achieved, a behavior probably related to a change in strain or in growth mode. ${ }^{34}$

CL hyperspectral imaging ${ }^{26}$ was used to determine the photon emission characteristics of the thick InGaN layers formed on the different facets of the NRs and to estimate, assuming the InGaN layer is thick enough to be relaxed, the $\mathrm{InN}$ mole fraction, using the emission energy as a function of composition. ${ }^{39,40}$ The estimated InGaN layer widths were sufficiently large that the quantum confinement effect is negligible, so that the photon emission energy provides a good estimate of the bandgap, hence composition. Hyperspectral CL measures a full spectrum from each point on the surface scanned by the electron beam. In this work, CL spectra were collected at room temperature for a beam energy of $5 \mathrm{keV}$ and step size of $25 \mathrm{~nm}$, to create $200 \times 200$ pixel maps. Figures 4(a)-4(c) show real-color images that have been determined from the chromaticity coordinates of the collected spectra and thereby illustrate the impact of the growth conditions on the obtained InGaN layer.

The CL maps in Figs. 4(a)-4(c) show for each sample the various facet classes have a different real color, caused by different InGaN composition and facet-dependent InN fraction incorporation rates. Corresponding spectra extracted from the same hyperspectral datasets are shown in Fig. 4(d) for the crystal planes considered. The spectra were taken from representative areas averaging $4 \times 4$ pixels from each facet. For all samples, the residual (0001) plane emits in the range of 2.05 to $2.28 \mathrm{eV}$, with different relative intensities from sample to sample.

On the sample grown at $750^{\circ} \mathrm{C}$ and 300 mbar [Fig. 4(a)], the $\{10-10\} m$-plane displays emission mainly at $2.83 \mathrm{eV}$. Compared to the other samples, the residual $\{11-20\} a$-plane is much
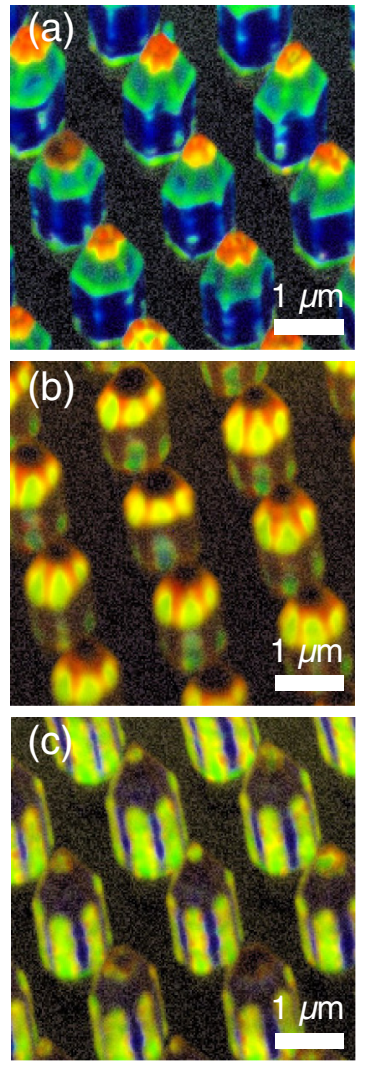

(d)

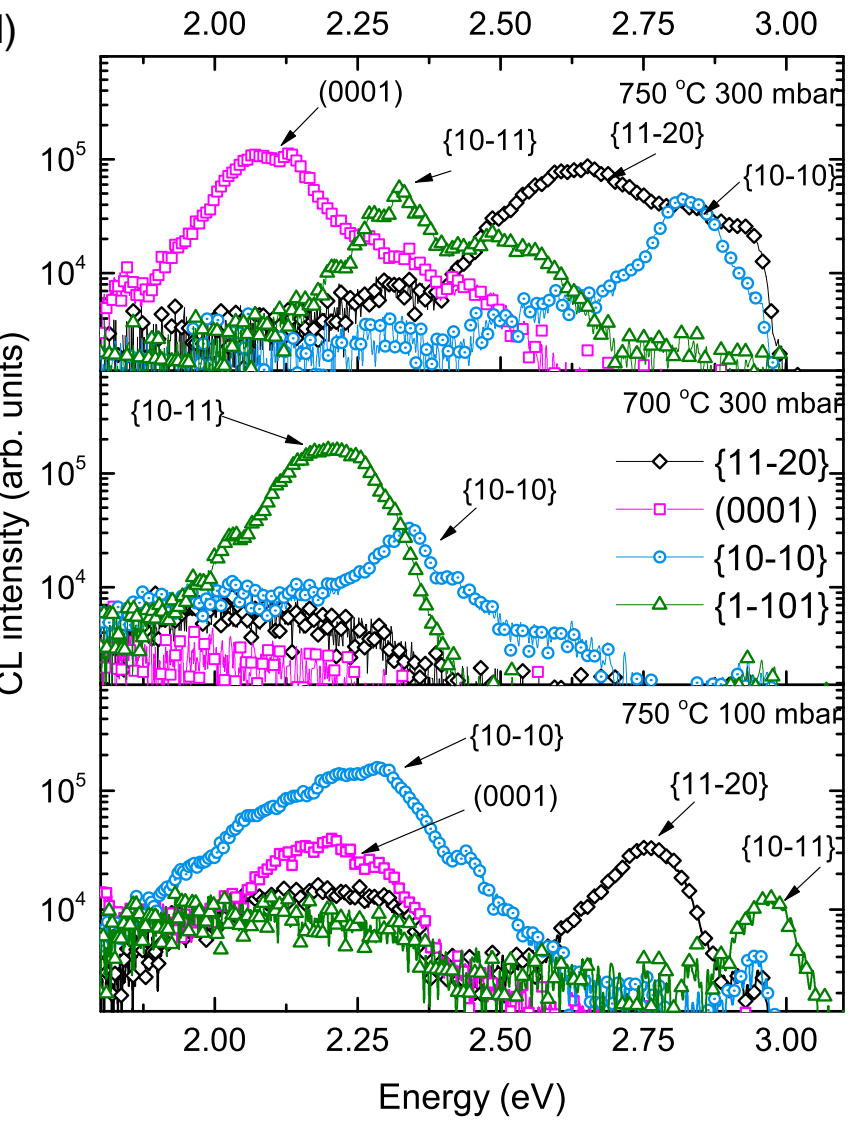

Fig. 4 Real-color images and corresponding CL spectra of samples with different growth conditions. (a) $750^{\circ} \mathrm{C} 300 \mathrm{mbar}$ with dissimilar emission from every facet, (b) $700^{\circ} \mathrm{C} 300 \mathrm{mbar}$ with main emission from the $\{1-101\}$ facets, (c) $750^{\circ} \mathrm{C} 100$ mbar with high intensity emission from the $m$-plane facets, and (d) spectra collected from a representative area of $4 \times 4$ pixels of each facet. 
smaller due to its faster growth rate relative to $\{10-10\} m$-planes and has an emission centered on $2.65 \mathrm{eV}$, with a high energy shoulder at $2.83 \mathrm{eV}$ due to simultaneous emission from the nearby $m$-planes. The $\{1-101\}$ semipolar facets exhibit two emission peaks, changing emission color from the middle of the facet toward its edges. The facet center emits at $2.49 \mathrm{eV}$, whereas the most intense emission originates from the apices, at $2.33 \mathrm{eV}$. The latter is caused by either a reduction in strain at the apices, or by increased indium atom incorporation in the localized regions, or both.

For the $700^{\circ} \mathrm{C}$ InGaN growth, no emission was identified on the rough $a$-plane, possibly due to surface states introduced by roughening, acting as paths for nonradiative recombination ${ }^{41}$ at high indium concentrations. ${ }^{42}$ There were areas with emission peaks from the $\{10-10\}$ facets at $2.35 \mathrm{eV}, 2.62 \mathrm{eV}$, and a weak emission at $2.95 \mathrm{eV}$. This is probably caused by a varying indium fraction incorporation on the more irregular surfaces of this sample, while the $2.95 \mathrm{eV}$ may originate from the first-grown region of the InGaN layer observable in the TEM image [see Fig. 3(d)]. The dominant emission for this sample originates on the $\{1-101\}$ semipolar facets and is centered at $2.20 \mathrm{eV}$. Overall, the decrease in temperature resulted in red-shifted emission from all the facets due to higher indium incorporation on all surfaces as a consequence of reduced desorption rate.

Figure 4(c), for the growth at $750^{\circ} \mathrm{C}$ and 100 mbar, shows a broad emission around $2.29 \mathrm{eV}$, which originates on the $\{10-10\}$ facets, with additional peaks at 2.43 and $2.95 \mathrm{eV}$, the latter related to a strained layer as well. The $\{11-20\}$ planes have a distinct emission at $2.75 \mathrm{eV}$. The $\{1-101\}$ semipolar planes emit at $2.97 \mathrm{eV}$ with a low intensity peak, and this emission intensity is relatively quenched by 1 order of magnitude with respect to the other crystal facets. There is a corresponding increase in the emission from the defect-related "yellow band." The incorporation of a larger number of point defects at this pressure could explain why the main band emission peak is quenched.

The relative CL emission peak intensities can give some general indications of the variations in crystal layer quality on the different facets with the growth parameters. For both $a$ - and $m$ nonpolar planes, higher CL intensities were obtained at higher temperature and lower pressure. By contrast, the emission intensity for the semipolar $\{1-101\}$ plane favors lower temperature and higher pressure, also leading to high $\mathrm{InN}$ fractions being incorporated. However, such conditions limit emission from the $m$-plane [Fig. 4(b)].

\section{Discussion}

The results confirm that the InGaN growth rates and InN mole fraction are strongly dependent on the crystal plane orientation and the growth parameters. The growth parameters can be used to alter the relative growth rates between the $m$ - and $a$-plane, leading to different extents of the \{11-20\} facet, when starting from identical faceted GaN NRs. Roughness on the crystal facets, caused by either unstrained InGaN growth resulting from a 3-D growth mode or rapid $\mathrm{InN}$ incorporation related to the short diffusion length of In, causes local variations of the indium mole fraction and a likely widening of the CL emission spectra.

The decrease of the growth temperature from $750^{\circ} \mathrm{C}$ to $700^{\circ} \mathrm{C}$ led to an increase of the $\mathrm{InN}$ mole fraction, estimated by CL to be from $13 \%$ to $24 \%$ on the $\{10-10\}$ plane, and from $20-25 \%$ to $25-30 \%$ on the $\{1-101\}$ plane. The difference in contrast along the [10-10] direction observed in TEM for the $700^{\circ} \mathrm{C}$ sample [see Fig. 3(d)], from a dark InGaN layer to a brighter one, indicates a possible increase of the InN mole fraction ${ }^{43}$ after the growth of an initial pseudomorphic InGaN layer.

As can be seen in Table 2, at $300 \mathrm{mbar}$, the average InN mole fraction was found to be higher on the $\{11-20\} a$-plane (17\%), and the $\{1-101\}$ plane (20-25\%), than for the $\{10-10\} m$-plane, where the indium composition is $13 \%$. This result indicates that, despite the thick shell allowing for unstrained growth, this set of growth conditions still limits the $\mathrm{InN}$ fraction incorporation efficiency.

The reduction of pressure from 300 to 100 mbar has had a large impact on the growth of all crystal planes. Use of 100 mbar pressure and $750^{\circ} \mathrm{C}$ growth temperature caused a change in the InN mole fraction between the nonpolar planes; specifically, a greater indium composition 
Table 2 Correlation of observed $\mathrm{CL}$ peak emission energy and $\mathrm{InN}$ mole fraction, as determined by the composition dependence of the bandgap.

\begin{tabular}{lccccccccc}
\hline \hline Sample & \multicolumn{3}{c}{$750^{\circ} \mathrm{C} 300 \mathrm{mbar}$} & \multicolumn{2}{c}{$700^{\circ} \mathrm{C} 300 \mathrm{mbar}$} & \multicolumn{3}{c}{$750^{\circ} \mathrm{C} 100 \mathrm{mbar}$} \\
\hline Plane & $\{10-10\}$ & $\{11-20\}$ & $\{1-101\}$ & $\{10-10\}$ & $\{11-20\}$ & $\{1-101\}$ & $\{10-10\}$ & $\{11-20\}$ & $\{1-101\}$ \\
Peak (eV) & 2.83 & 2.65 & 2.33 to 2.49 & 2.35 to 2.95 & - & 2.20 & 2.29 to 2.95 & 2.76 & 2.97 \\
$\operatorname{InN}(\%)$ & 13 & 17 & 25 to 20 & 24 to $14^{\mathrm{a}}$ & - & 27 & 25 to $14^{\mathrm{a}}$ & 15 & $13^{\mathrm{a}}$ \\
\hline \hline
\end{tabular}

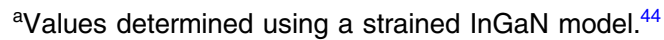

resulted on the $m$-planes (25\%) than on the $a$-planes (15\%) or on the semipolar $\{1-101\}$ planes ( $10 \%$ or more accurately $13 \%$, if an unstrained InGaN model ${ }^{44}$ is considered). This was unexpected as the growth rate was low on this sample (see Table 1), and both low pressure and high temperature cause high diffusion lengths for the indium atoms. However, from work on $c$-plane ${ }^{45,46}$ and $a$-plane ${ }^{47}$ planar thin films, it was found that low pressure can increase indium incorporation. This was suggested to be caused by the suppression of indium desorption with enhanced gas mass transfer rates of precursor species through the boundary layer, ${ }^{45}$ as the diffusion in gases varies inversely proportional with pressure. ${ }^{48}$ The measurements reported here show that low pressure encourages high $\mathrm{InN}$ incorporation efficiency on the $m$-plane, thus allowing InGaN layers with high $\mathrm{InN}$ mole fraction to be grown.

\section{Conclusion}

A GaN template of vertically aligned NRs with (0001), $\{10-10\},\{11-20\}$ and $\{1-101\}$ facets was fabricated to study the effect of pressure and temperature on the growth rates and emission characteristics of thick ( $\sim 40$ to $80 \mathrm{~nm}$ ) InGaN layers for core-shell LEDs. The growth rates of the nonpolar planes were determined from SEM images and correlated with TEM analysis. The InGaN growth rates on the nonpolar planes varied from 1.3 to $2.8 \mathrm{~nm} / \mathrm{min}$, depending on growth temperature and pressure. Each growth led to a different set of emission peaks, i.e., a different $\mathrm{InN}$ fraction was incorporated on each facet. The $\mathrm{InN}$ mole fraction incorporated on each crystal facet was estimated by comparing the emission peak measured by CL hyperspectral imaging with previously published data. ${ }^{39,40,44}$ The study revealed that the reduction of pressure to 100 mbar led to a more efficient $\mathrm{InN}$ mole fraction integration on the $\{10-10\}$ $m$-plane, increasing it from $13 \%$ to $25 \%$. This may be used to obtain green-yellow coreshell LEDs with a high InN mole fraction based on emission from the $\{10-10\} \mathrm{m}$-planes. The study has shown how growth parameters can control the relative crystal facet growth rates and $\mathrm{InN}$ mole fraction. This understanding can guide design for core-shell LEDs.

\section{Acknowledgments}

The authors would like to thank OSRAM Opto Semiconductors GmbH for the provision of the $\mathrm{GaN} /$ silicon templates and acknowledge financial support from the European Union (FP7 Contract No.: 228999, "SMASH") and the EPSRC, UK via Grant No. EP/I012591/1 "Lighting the Future." We gratefully acknowledge the Leeds EPSRC Nanoscience and Nanotechnology Facility (Grant No. EP/K023853/1) and Dr. Michael Ward for the focused ion beam (FIB) sample preparation. This publication is supported by multiple datasets, which are openly available at locations cited in the reference section.

\section{References}

1. A. E. Romanov et al., "Strain-induced polarization in wurtzite III-nitride semipolar layers," J. Appl. Phys. 100(2), 023522 (2006).

2. R. M. Farrell et al., "Materials and growth issues for high-performance nonpolar and semipolar light-emitting devices," Semicond. Sci. Technol. 27(2), 024001 (2012). 
3. Y. J. Hong et al., "Visible-color-tunable light-emitting diodes," Adv. Mater. 23(29), 32843288 (2011).

4. J.-R. Chang et al., "Fabrication and luminescent properties of core-shell InGaN/GaN multiple quantum wells on GaN nanopillars," Appl. Phys. Lett. 100(26), 261103 (2012).

5. T.-W. Yeh et al., "InGaN/GaN multiple quantum wells grown on nonpolar facets of vertical GaN nanorod arrays," Nano Lett. 12(6), 3257-3262 (2012).

6. K. Kishino, H. Sekiguchi, and A. Kikuchi, "Improved Ti-mask selective-area growth (SAG) by rf-plasma-assisted molecular beam epitaxy demonstrating extremely uniform GaN nanocolumn arrays," J. Cryst. Growth 311(7), 2063-2068 (2009).

7. A. Waag et al., "The nanorod approach: GaN NanoLEDs for solid state lighting," Phys. Status Solidi 8(7-8), 2296-2301 (2011).

8. S. D. Hersee, X. Sun, and X. Wang, "The controlled growth of GaN nanowires," Nano Lett. 6(8), 1808-1811 (2006).

9. T. Tang et al., "Nitride nanocolumns for the development of light-emitting diode," IEEE Trans. Electron. Dev. 57(1), 71-78 (2010).

10. W. Bergbauer et al., "Continuous-flux MOVPE growth of position-controlled N-face GaN nanorods and embedded InGaN quantum wells," Nanotechnology 21(30), 305201 (2010).

11. K. Tomioka et al., "III-V nanowires on Si substrate: selective-area growth and device applications," IEEE J. Sel. Top. Quantum Electron. 17(4), 1112-1129 (2011).

12. C.-H. Liao et al., "Cross-sectional sizes and emission wavelengths of regularly patterned GaN and core-shell InGaN/GaN quantum-well nanorod arrays," J. Appl. Phys. 113(5), 054315 (2013).

13. K. Choi, M. Arita, and Y. Arakawa, "Selective-area growth of thin GaN nanowires by MOCVD," J. Cryst. Growth 357, 58-61 (2012).

14. P.-M. Coulon et al., "Selective area growth of Ga-polar GaN nanowire arrays by continuousflow MOVPE: a systematic study on the effect of growth conditions on the array properties," Phys. Status Solidi 252(5), 1096-1103 (2015).

15. P. A. Shields et al., "Fabrication and properties of etched GaN nanorods," Phys. Status Solidi 9(3-4), 631-634 (2012).

16. S. Krylyuk et al., "Large-area GaN n-core/p-shell arrays fabricated using top-down etching and selective epitaxial overgrowth," Appl. Phys. Lett. 101(24), 241119 (2012).

17. E. D. Le Boulbar et al., "Facet recovery and light emission from GaN/InGaN/GaN coreshell structures grown by metal organic vapour phase epitaxy on etched $\mathrm{GaN}$ nanorod arrays," J. Appl. Phys. 114(9), 094302 (2013).

18. M. Hugues et al., "Strain evolution in GaN nanowires: From free-surface objects to coalesced templates," J. Appl. Phys. 114(8), 084307 (2013).

19. P. A. Shields et al., "Nanopendeo coalescence overgrowth of GaN on etched nanorod array," Phys. Status Solidi Curr. Top. Solid State Phys. 8(7-8), 2334-2336 (2011).

20. J. R. Riley et al., "Three-dimensional mapping of quantum wells in a $\mathrm{GaN} / \mathrm{InGaN}$," Nano Lett. 13, 4317-4325 (2013).

21. C. J. Lewins et al., "Strong photonic crystal behavior in regular arrays of core-shell and quantum disc InGaN/GaN nanorod light-emitting diodes," J. Appl. Phys. 116(4), 044305 (2014).

22. S. Li and A. Waag, "GaN based nanorods for solid state lighting," J. Appl. Phys. 111(7), 071101 (2012).

23. P. A. Shields and D. W. E. Allsopp, "Nanoimprint lithography resist profile inversion for lift-off applications," Microelectron. Eng. 88(9), 3011-3014 (2011).

24. I. Gîrgel et al., "Dataset for investigation of InGaN facet-dependent non-polar growth rates and composition for core-shell LEDs," http://doi.org/10.15125/BATH-00172 (25 February 2016).

25. H. P. D. Schenk et al., "Indium incorporation above $800^{\circ} \mathrm{C}$ during metalorganic vapor phase epitaxy of InGaN," Appl. Phys. Lett. 75(17), 2587 (1999).

26. P. R. Edwards and R. W. Martin, "Cathodoluminescence imaging and spectroscopy of InGaN/GaN core-shell nanostructures," http://dx.doi.org/10.15129/53c3bd47-91d8-40cea6ac-98e46b893588 (25 February 2016). 
Gîrgel et al.: Investigation of indium gallium nitride facet-dependent nonpolar growth rates...

27. N. A. Fichtenbaum et al., "Metalorganic chemical vapor deposition regrowth of InGaN and GaN on N-polar pillar and stripe nanostructures," Jpn. J. Appl. Phys. 46(10), L230-L233 (2007).

28. C. Liu et al., "Light emission from InGaN quantum wells grown on the facets of closely spaced GaN nano-pyramids formed by nano-imprinting," Appl. Phys. Express 2(12), 121002 (2009).

29. C. Miao et al., "Growth of InGaN/GaN multiple quantum wells on size-controllable nanopyramid arrays," Jpn. J. Appl. Phys. 53, 030306 (2014).

30. B. Leung et al., "Using the kinetic Wulff plot to design and control nonpolar and semipolar GaN heteroepitaxy," Semicond. Sci. Technol. 27(2), 024005 (2012).

31. S. Pereira et al., "Structural and optical properties of InGaN/GaN layers close to the critical layer thickness," Appl. Phys. Lett. 81(7), 1207 (2002).

32. M. Leyer et al., "The critical thickness of InGaN on (0001)GaN," J. Cryst. Growth 310(23), 4913-4915 (2008).

33. B. N. Bryant et al., "Quasi-equilibrium crystal shapes and kinetic Wulff plots for gallium nitride grown by hydride vapor phase epitaxy," J. Cryst. Growth 369, 14-20 (2013).

34. Q. Sun et al., "Understanding nonpolar GaN growth through kinetic Wulff plots," J. Appl. Phys. 104(9), 093523 (2008).

35. J. E. Northrup and J. Neugebauer, "Theory of GaN (10-10) and (11-20) surfaces," Phys. Rev. B 53(16), R10477-10480 (1996).

36. J. Neugebauer, "Ab initio analysis of surface structure and adatom kinetics of group-III nitrides," Phys. Status Solidi 227(1), 93-114 (2001).

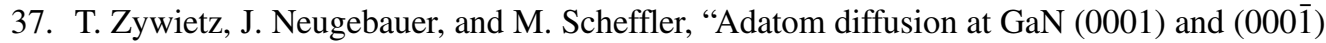
surfaces," Appl. Phys. Lett. 73(4), 487 (1998).

38. H. K. Cho et al., "Influence of growth temperature and reactor pressure on microstructural and optical properties of InAlGaN quaternary epilayers," J. Cryst. Growth 267(1-2), 67-73 (2004)

39. V. Y. Davydov et al., "Band gap of hexagonal InN and InGaN alloys," Phys. Status Solidi 234(3), 787-795 (2002).

40. K. P. O'Donnell et al., "The composition dependence of the Inx Ga1-x N bandgap," J. Cryst. Growth 269(1), 100-105 (2004).

41. C. Hahn et al. "Effect of thermal annealing in ammonia on the properties of InGaN nanowires with different indium concentrations," J. Phys. Chem. C 117(7), 3627-3634 (2013).

42. T. Langer et al., "Origin of the 'green gap': increasing nonroadiative recombination in indium-rich GaInN/GaN quantum well structures," Phys. Status Solidi 8(7-8), 21702172 (2011).

43. Q. Li and G. T. Wang, "Strain influenced indium composition distribution in GaN/InGaN core-shell nanowires," Appl. Phys. Lett. 97(18), 181107 (2010).

44. G. Orsal et al., "Bandgap energy bowing parameter of strained and relaxed InGaN layers," Opt. Mater. Express 4(5), 1030 (2014).

45. D. Kim et al., "Effect of growth pressure on indium incorporation during the growth of InGaN by MOCVD," J. Electron. Mater. 30(2), 99-102 (2001).

46. R. A. Oliver et al., "The influence of ammonia on the growth mode in InGaN/GaN heteroepitaxy," J. Cryst. Growth 272(1-4), 393-399 (2004).

47. K.-M. Song and J. Park, "Effects of the growth pressure of a-plane InGaN/GaN multi-quantum wells on the optical performance of light-emitting diodes," Semicond. Sci. Technol. 28(1), 015010 (2013).

48. M. Ohring, "Chemical vapor deposition," Chapter 6 in Materials Science of Thin Films Deposition and Structure, 2nd Ed., p. 296, Academic Press, London (1992).

Ionut Girgel is a $\mathrm{PhD}$ student in the Department of Electronic and Electrical Engineering at the University of Bath. He received his MSc in condensed matter physics and his BSc in applied physics from Ovidius University of Constanta, Romania, in 2008 and 2009, respectively. His $\mathrm{PhD}$ research is in the area of core-shell InGaN/GaN devices. He is a member of SPIE.

Paul R. Edwards is a senior research fellow in the Department of Physics at the University of Strathclyde. He received his BSc and $\mathrm{PhD}$ degrees from Imperial College London and the 
University of Durham in 1995 and 1999, respectively. He has authored 120 journal and conference papers on the optical and electron beam characterization of compound semiconductors.

Emmanuel Le Boulbar is a research officer in the Department of Electrical and Electronic Engineering at the University of Bath. He received his DPhil degree in material science from the University of Orleans, France, in 2010. His current research interests include the growth and fabrication of III-nitride nanostructures for optoelectronic and water-splitting applications.

Pierre-Marie Coulon is a research assistant in the Department of Electronic and Electrical Engineering at the University of Bath. He received MPhys and DPhil degrees in physics from the University of Nice Sophia-Antipolis in 2010 and 2014, respectively. His research interest is in the growth and characterization of III-nitride devices mainly for optoelectronic applications, with a particular focus on growth mechanisms within nanostructures.

Suman-Lata Sahonta obtained her $\mathrm{PhD}$ in electron beam analysis of ultra-wide band gap nitride materials at the University of Bristol. She is currently a postdoctoral fellow in the Cambridge Centre for Gallium Nitride at the University of Cambridge.

Duncan W. E. Allsopp received his BSc degree in physics and $\mathrm{PhD}$ degrees from the University of Sheffield, in 1971 and 1977, respectively. Since 1999, he has been at the University of Bath, United Kingdom, where he leads the III-nitride research group. He is author and coauthor of over 230 papers on semiconductor materials, devices, and microsystems. He is a past Royal Academy of Engineering-Leverhulme Trust Senior Research Fellow.

Robert W. Martin is a professor of experimental physics in the Department of Physics at Strathclyde University in Glasgow, United Kingdom. He received his MA and DPhil degrees in physics from the University of Oxford in 1988 and 1992, respectively. He has published over 240 papers on the properties of semiconductor and other advanced materials, in particular, the III-nitrides. He is a fellow of the Institute of Physics.

Colin J. Humphreys is a professor and director of research in the Department of Materials Science and Metallurgy at the University of Cambridge. He has published over 600 papers on electron microscopy, gallium nitride and other materials and device structures. He is a fellow of the Royal Society and of the Royal Academy of Engineering.

Philip A. Shields is a lecturer (assistant professor) in the Department of Electronic and Electrical Engineering at the University of Bath. He received his MPhys and DPhil degrees in physics from the University of Oxford, United Kingdom, in 1997 and 2001, respectively. His research interest is in the growth and fabrication of III-nitride devices mainly for optoelectronic applications, with a particular focus on the use of nanostructures to improve device performance. 